\title{
Postsurgical Stage II Hepatoblastoma
}

National Cancer Institute

\section{Source}

National Cancer Institute. Postsurgical Stage II Hepatoblastoma. NCI Thesaurus. Code C7145.

No metastases, tumor grossly resected with microscopic residual disease (i.e., positive margins); or tumor rupture, or tumor spill at the time of surgery. (from PDQ 2004) 